\title{
Saúde em vista: uma análise da Atenção Primária à Saúde em áreas ribeirinhas e rurais amazônicas
}

\author{
Health in sight: an analysis of Primary Health Care \\ in riverside and rural Amazon areas
}

Rodrigo Tobias de Sousa Lima (https://orcid.org/0000-0002-4098-5276) ${ }^{1}$

Tiótrefis Gomes Fernandes (https://orcid.org/0000-0002-8563-9529) ${ }^{2}$

Paulo Jorge Alves Martins Júnior (https://orcid.org/0000-0002-2688-6124) ${ }^{3}$

Cleudecir Siqueira Portela (https://orcid.org/0000-0002-1616-0055) ${ }^{4}$

James Dean Oliveira dos Santos Junior (https://orcid.org/0000-0002-0627-6126) ${ }^{5}$

Júlio Cesar Schweickardt (https://orcid.org/0000-0002-8349-3482) ${ }^{1}$

${ }^{1}$ Instituto Leônidas e Maria Deane (ILMD/Fiocruz

Amazônia), Laboratório de História, Políticas Públicas e Saúde na Amazônia (LAHPSA). R. Terezina 476, Adrianópolis. 69065001 Manaus AM Brasil. tobiasrodrigo@gmail.com ${ }^{2}$ Faculdade de Educação Física e Fisioterapia, Universidade Federal do Amazonas (UFAM).

Manaus AM Brasil.

${ }^{3}$ Graduação em Psicologia,

UFAM. Manaus AM Brasil.

${ }^{4}$ Departamento de Atenção

Básica e Ações Estratégicas,

Secretaria de Estado de

Saúde do Amazonas.

Manaus AM Brasil.

${ }^{5}$ Departamento de

Estatística, UFAM. Manaus

AM Brasil.

\begin{abstract}
The National Primary Care Policy has provided a significant change in terms of health actions in the riverside territory, establishing health teams and equipment. The Riverside and Fluvial Family Health teams for the Legal Amazon and Pantanal areas have been innovative regarding the integral care of the forest populations. This study aims to analyze primary health care in the rural and riverside context in the Amazon territory, based on the production of individual and collective actions by health teams, as well as their performance through services offered in the municipalities. The study is cross-sectional, with a descriptive-analytical design and quantitative approach, and analyzes the coverage of primary health care actions of family health strategy teams in urban, rural and riverside areas of eight municipalities located in the state of Amazonas. The results showed that there was an increase in primary care coverage in almost all analyzed municipalities, indicating that techno-assistance models such as the riverside and fluvial teams resulted in the inclusion of a population that is scattered over large areas of the territory of municipalities. A health policy that promotes equity results in changes and alterations in the ways of life and health status of the Amazonian populations. Key words Primary Health Care, Rural Health Services, Patient Care Team, Amazonas
\end{abstract}

Resumo A Política Nacional de Atenção Básica proporcionou uma mudança significativa no que se refere às ações da saúde no território ribeirinho, criando equipes e equipamentos de saúde. As equipes de Saúde Ribeirinha e Saúde Fluvial para as áreas da Amazônia Legal e Pantanal foram inovadoras na atenção integral das populações da floresta. Esse estudo tem como objetivo analisar a atenção primária em saúde no contexto rural e ribeirinha no território amazônico, a partir da produção de ações individuais e coletivas das equipes de saúde, bem como o seu desempenho por meio de serviços ofertados nos municípios. $O$ estudo é transversal, com delineamento descritivo -analítico e abordagem quantitativa, analisando a cobertura das ações de atenção básica de equipes da estratégia saúde da família em áreas urbanas, rurais e ribeirinhas de oito municípios situados no estado do Amazonas. Os resultados mostraram que houve aumento da cobertura da atenção básica em quase todos os municípios analisados, indicando que modelos tecnoassistenciais como das equipes ribeirinhas e fluviais produziram a inclusão de uma população que está dispersa em longas áreas do território dos municípios. Uma política de saúde com equidade produz mudanças e transformações nos modos de vida e nas condições de saúde das populações amazônicas.

Palavras-chave Atenção Primária em Saúde, Equipes de Saúde, Serviços de saúde rural, Amazônia 


\section{Introdução}

A Atenção Básica, também denominada Atenção Primária à Saúde ${ }^{1-3}$, fundamenta-se como aposta central do Sistema Único de Saúde (SUS). A Política Nacional de Atenção Básica (PNAB) busca a proteção social, a universalização do cuidado e, sobretudo, a produção de respostas às necessidades de saúde e acesso aos seus serviços de forma equitativa e integral para as populações, inclusive na Amazônia Brasileira ${ }^{2,4}$.

A PNAB proporcionou uma mudança significativa no que se refere às ações da Atenção Básica $(\mathrm{AB})$ na região, criando equipes e equipamentos de saúde ajustadas e direcionadas para as populações que vivem na Amazônia. Desse modo, surge um novo modelo de trabalho e de atenção através das equipes de Saúde Ribeirinha e Saúde Fluvial para as áreas da Amazônia Legal e Pantanal. Essas novas abordagens viabilizaram outro modelo tecnoassitencial que dialogasse com o território e suas populações ${ }^{5}$. A Unidade Básica de Saúde Fluvial (UBSF) e as Equipes de Saúde Ribeirinha $(\mathrm{eSFR})^{3}$ criaram o acesso à uma população de floresta que era historicamente marginalizada do processo de cuidado.

Em 2006 a primeira edição da PNAB, através da Portaria nº 648, de 28 de março de 2006, não contemplava as Equipes de Saúde da Família Ribeirinhas ou Fluviais. Apenas com a publicação da Portaria no 2.191, de 3 de agosto de 2010, é que se institui critérios diferenciados para a implantação, financiamento e manutenção destas equipes, que são destinadas ao atendimento de populações ribeirinhas na Amazônia Legal e Mato Grosso do Sul. A segunda edição da PNAB, através da Portaria no 2.488 , de 21 de outubro de $2011^{1}$, trouxe novos critérios para o planejamento das equipes e na terceira PNAB, Portaria no 2.436 , de 21 de setembro de $2017^{3}$, houve a revisão das diretrizes para a organização da atenção básica.

O presente estudo trata do território do estado do Amazonas, por ter uma extensa rede hidrográfica de mais de 6 mil quilômetros de rios navegáveis com diferentes tipologias (águas claras e escuras) e bioma ${ }^{6}$, uma grande diversidade étnica-cultural, baixa densidade populacional e com o maior número de equipes de saúde da família fluvial e ribeirinhas dos estados da região norte ${ }^{7}$. Esses fatores interferem na gestão dos serviços de saúde, especialmente no acesso da atenção básica pelas populações quilombolas, ribeirinhas e indígenas. Pelas condições de vida e os modos de conceber o território, destaca-se que o sistema de transporte fluvial nas áreas rurais e ribeirinhas está limitado pelas distâncias, pelas calhas dos rios e pelo regime das águas. Assim, o cronograma da atenção também deve considerar essa territorialidade, que também sofre influência dos aspectos econômicos, culturais e mediados pela sazonalidade dos rios. O território amazonense pode ser analisado a partir da categoria do território líquido ${ }^{8,9}$ que tem relação com os tipos de política públicas de saúde a serem desenvolvidas na Amazônia.

Diante do exposto, o entendimento sobre o desenvolvimento e a implementação das políticas públicas específicas para o território amazônico nos ajudam nas análises sobre o acesso aos serviços de saúde pela população considerando o princípio de equidade do SUS. Realizar abordagens que produzam a visibilidade da atenção primária em áreas rurais e ribeirinhas amazônicas, especialmente o trabalho das equipes de saúde, contribuem para o fortalecimento desse modelo. As Unidades Básicas de Saúde da Família (UBSF) urbanas como as UBS Fluviais e Ribeirinhas têm garantido o acesso aos serviços de saúde para as populações em áreas remotas e de difícil acesso. Desta forma, o presente estudo teve como objetivo analisar a atenção primária rural e ribeirinha no território amazônico, a partir da produção de ações individuais e coletivas das equipes de saúde, bem como o seu desempenho por meio de serviços ofertados em municípios do estado do Amazonas.

\section{Metodologia}

O Amazonas é o maior estado da federação, sendo entrecortado por uma extensa rede hidrográfica, formada por rios, lagos, paranás e igarapés. $\mathrm{O}$ estado ainda tem preservado as suas florestas através de políticas ambientais e socioeconômicas através de parques, reservas, reservas extrativistas, terras indígenas, com exceção a região sul do Estado onde o agronegócio está se expandindo desde a década de 1970. A densidade demográfica é baixa, ficando em torno de 2,23 hab $/ \mathrm{km}^{2}$, tendo parte da população vivendo em comunidades localizadas nas margens das águas ${ }^{6}$. Um fator que impacta nos serviços de saúde, são as grandes distâncias entre as comunidades e a sede do município, pois acaba por concentrar, em sua grande maioria, as ações e serviços de saúde na sede dos municípios.

O presente estudo abordou a realidade da atenção básica de oito municípios, sendo esses: 
Barreirinha, Borba, Careiro da Várzea, Humaitá, Nhamundá, Novo Aripuanã, Tefé e Urucurituba (Figura 1). Foram utilizados como critérios de inclusão os municípios que apresentaram: (a) pelo menos uma equipe de estratégia de saúde da família rural, ribeirinha e urbana; (b) pelo menos uma unidade básica de saúde fluvial em operação durante o ano de 2019; (c) produção de atendimentos individuais e visitas domiciliares enviados mensalmente ao Ministério da Saúde (MS). A capital Manaus foi excluída do estudo por se tratar de uma localidade que compreende mais da metade da população do estado, com alta concentração urbana e baixa relação com o rural, representa um caso de análise específico nos estudos sobre análises de políticas de saúde na região amazônica. Além disso, foram excluídos municípios elegíveis que não informou suas produções nos sistemas oficiais de informação por mais de uma competência.

Foi realizado um estudo transversal, com delineamento descritivo-analítico e abordagem quantitativa, que analisou a cobertura das ações de atenção básica de equipes da estratégia saúde da família tanto em áreas urbanas, quanto em áreas rurais e ribeirinhas de municípios situados no estado do Amazonas.
Os dados utilizados estão disponíveis no site do Ministério da Saúde e são oriundos do Sistema de Informação em Saúde para a Atenção Básica (SISAB), consultando as informações de produção, como parte da estratégia e-SUS $\mathrm{AB}$, sendo, para o estudo: a Coleta de Dados Simplificada (CDS) ou Prontuário Eletrônico do Cidadão (PEC). Esses registros foram gerados de acordo com o trabalho dos profissionais da Atenção Básica - equipes da Estratégia Saúde da Família que são submetidos a um processo de validação, considerando: se há duplicidade do envio de dados e limpeza do excesso de dados; a data do envio de dados; e mediante confirmação da informação que verifica se o número do estabelecimento no Cadastro Nacional de Estabelecimentos em Saúde (CNES), o número do Identificador Nacional de Equipes (INE), o número do Cartão Nacional de Saúde (CNS) e o Código Brasileiro de Ocupações $(\mathrm{CBO})$ do profissional estão válidos, checando os dados disponíveis na base do Sistema do CNES referente à competência da produção e se existe vínculo único entre eles ${ }^{10}$.

Ainda no SISAB foram coletados dados das produções de atividades coletivas e de produção ambulatorial individual e visitas domiciliares, segundo o tipo de equipe (INE da equipe de saúde 
da família; equipe de saúde rural; equipe de saúde ribeirinha) com vínculo a Unidade de Saúde da Família, Unidade de Saúde da Família Fluvial e seu respectivo CNES; o tipo de profissional (medicina, enfermagem, agentes comunitários de saúde), baseado na análise de informações disponíveis nas bases de dados do MS e IBGE ${ }^{11}$.

Para avaliar a produção de serviços, foram variáveis de interesse os atendimentos individuais e as ações coletivas, que são o conjunto de ações dos membros da equipe (INE) por competência, vinculados a um estabelecimento de saúde (CNES) ao longo do ano de 2019. Também foram calculadas razões dos atendimentos e das ações coletivas, para efeitos de comparação, e foi utilizado o denominador que correspondeu a população cadastrada no SISAB, segundo o tipo de equipe.

Para avaliar o desempenho da atenção básica, foram calculadas médias dos indicadores do Programa Previne Brasil ${ }^{12}$ por tipo de equipe de saúde da família, para o ano de 2019: a) proporção de gestantes com pelo menos 6 (seis) consultas pré-natal realizadas, sendo a primeira até a $20^{\mathrm{a}}$ semana de gestação; b) proporção de gestantes com realização de exames para sífilis e HIV; c) proporção de gestantes com atendimento odontológico realizado; d) cobertura de exame citopatológico; e) proporção de pessoas hipertensas com Pressão Arterial (PA) aferida a cada 6 meses; f) proporção de diabéticos com solicitação de hemoglobina glicada. Quanto ao item "cobertura vacinal de Poliomielite inativada e de Pentavalente", os dados não estavam disponíveis.

Os municípios foram classificados de acordo com a tipologia determinada pelo IBGE. Características tais como densidade demográfica, localização em relação aos principais centros urbanos e tamanho da população são os critérios fundamentais da metodologia proposta para classificar as áreas urbanas e rurais no Brasil. A partir do cruzamento dessas variáveis, os municípios do estudo foram classificados em cinco tipos: urbano, intermediário adjacente, intermediário remoto, rural adjacente e rural remoto ${ }^{13}$. E para fins de análise, foram agregados os municípios que tinham características rurais em uma única classe.

Foram utilizadas planilhas Excel para montagem de banco de dados e, posteriormente, foi utilizado o software livre "R", versão 3.1.2 para efetiva análise. A análise estatística baseou-se em cálculo de médias das variáveis quantitativas e intervalos de 95\% de confiança (IC95\%) e o nível de significância adotado foi de $5 \%$. O gráfico de tendência foi construído, considerando a média das taxas de cobertura da atenção básica dos municípios do estudo por triênio no período de 12 anos. A pesquisa respeitou as diretrizes referentes à pesquisa envolvendo seres humanos, sendo aprovada no Comitê de Ética em Pesquisa.

\section{Resultados}

Dos 8 municípios que compõem a amostra, há 2 considerados urbanos pelo IBGE, únicos com população estimada acima de 50.000 habitantes para 2019 e com menores proporções de população rural em 2010, e, consequentemente, maiores quantidades de equipe de saúde da família (ESF) urbanas. O município de Careiro da Várzea foi o que possui maior quantidade de ESF ribeirinhas 8 , dado sua população ser composta em sua maioria por residentes da área rural. Em geral, as ESF de área rural (fluvial e ribeirinha) destes municípios foram responsáveis por cadastrar e acompanhar aproximadamente 60.000 pessoas em 2019 . E se considerada como base a população rural registrada em 2010, percebe-se a representação populacional rural da cobertura deste serviço em cada município, com destaque para quase a totalidade de cobertura em Tefé (Tabela 1).

A Figura 2 mostra que a maioria dos municípios aumentou sua cobertura de $A B$ nos 12 anos analisados, sobretudo no último triênio. Foi observado uma redução em Borba, que saiu de $100 \%$ de cobertura de $\mathrm{AB}$ de 2008-2010 para 77\% no último triênio, com diminuição a cada período, e em Novo Aripuanã, que, apesar do aumento da cobertura nos primeiros 9 anos, registrou forte queda em 2017-2019 e chegando a $64 \%$. O município de Urucurituba que manteve os $100 \%$ de cobertura de 2008 a 2019.

A Tabela 2 apresenta a produção mensal por tipo de equipe em cada tipo de município. No geral, percebeu-se que a quantidade total média das ações individuais e coletivas foram similares entre os tipos de equipe, e que há mais ações individuais nos municípios com características urbanas, em comparação àqueles rurais, diferença menos acentuada quando considerado a população cadastrada de cada tipo de equipe (razão). No total dos municípios, as ESF fluviais registraram menor capacidade de produção de ações individuais entre profissionais da medicina e enfermagem e ACS em relação aos outros tipos de ESF, quando considerada a população cadastrada. Os técnicos ou auxiliares de enfermagem geraram maior média de ações individuais entre ESF ribeirinhas do que entre ESF urbanas, independente do tipo de 


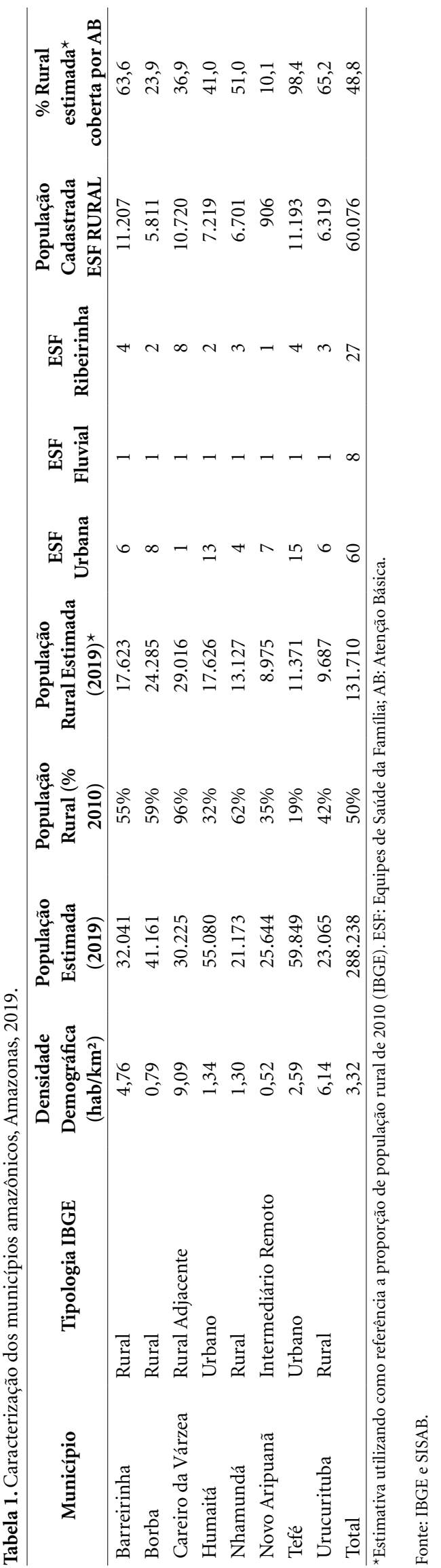

município, e do que ESF fluviais para municípios urbanos. Ressalta-se, ainda na Tabela 2, a baixa quantidade de ações coletivas, principalmente entre os profissionais médicos, os quais não registraram este tipo de ação em ESF fluviais.

Sobre o desempenho das equipes de $\mathrm{AB}$ ( $\mathrm{Ta}$ bela 3), no geral, apesar de maiores médias dos indicadores do Previne entre ESF urbanas, não há diferenças significativas em relação aos demais tipos de ESF. Foi observado, por outro lado, diferenças para proporção de 6 consultas de pré-natal, quando em comparação com ESF fluviais, e para proporção de diabéticos com hemoglobina glicada, em comparação aos demais tipos de ESF. No entanto, vale salientar que o baixo número de equipes rurais causa grande variabilidade da média, o que dificulta a demonstração de diferenças estatisticamente significativas.

\section{Discussão}

O presente estudo nos conduz à discussão sobre a avaliação de políticas públicas na Amazônia, especialmente no que diz respeito à melhoria de acesso das populações ribeirinhas aos cuidados da AB.

As equipes ribeirinhas e fluviais não necessariamente significaram um aumento da cobertura da atenção básica nos municípios, pois os mesmos já apresentavam altas taxas de cobertura com outros modelos de equipe, a partir dos critérios adotados pelo MS. No entanto, a possibilidade de diferenciar os tipos de equipe e diversificar os processos de trabalho, assim como ampliar o valor de custeio mensal, trouxe uma cobertura próxima às necessidades da população ribeirinha com respeito à integralidade e a continuidade do cuidado. Os resultados do estudo mostram que as equipes ribeirinhas e fluviais, declaradas pelos municípios, ainda são insuficientes para a cobertura efetiva desse território. Portanto, a discussão sobre a cobertura da atenção básica na Amazônia é objeto de discussão de gestores e pesquisadores, principalmente na mudança do cálculo e de regras de financiamento das equipes ${ }^{7,14}$. $\mathrm{O}$ aumento da cobertura só é possível com o financiamento tripartite ${ }^{15}$, que possibilite ampliar o número de equipes e diversificar os tipos, de acordo com a realidade de cada território. No entanto, a $\mathrm{PNAB}^{3}$ atual gerou uma diminuição de equipes ${ }^{14}$, como foi o caso de um município de Santarém, estado do Pará ${ }^{16}$.

A categoria de território líquido tem sido utilizada para dar um destaque às características do 


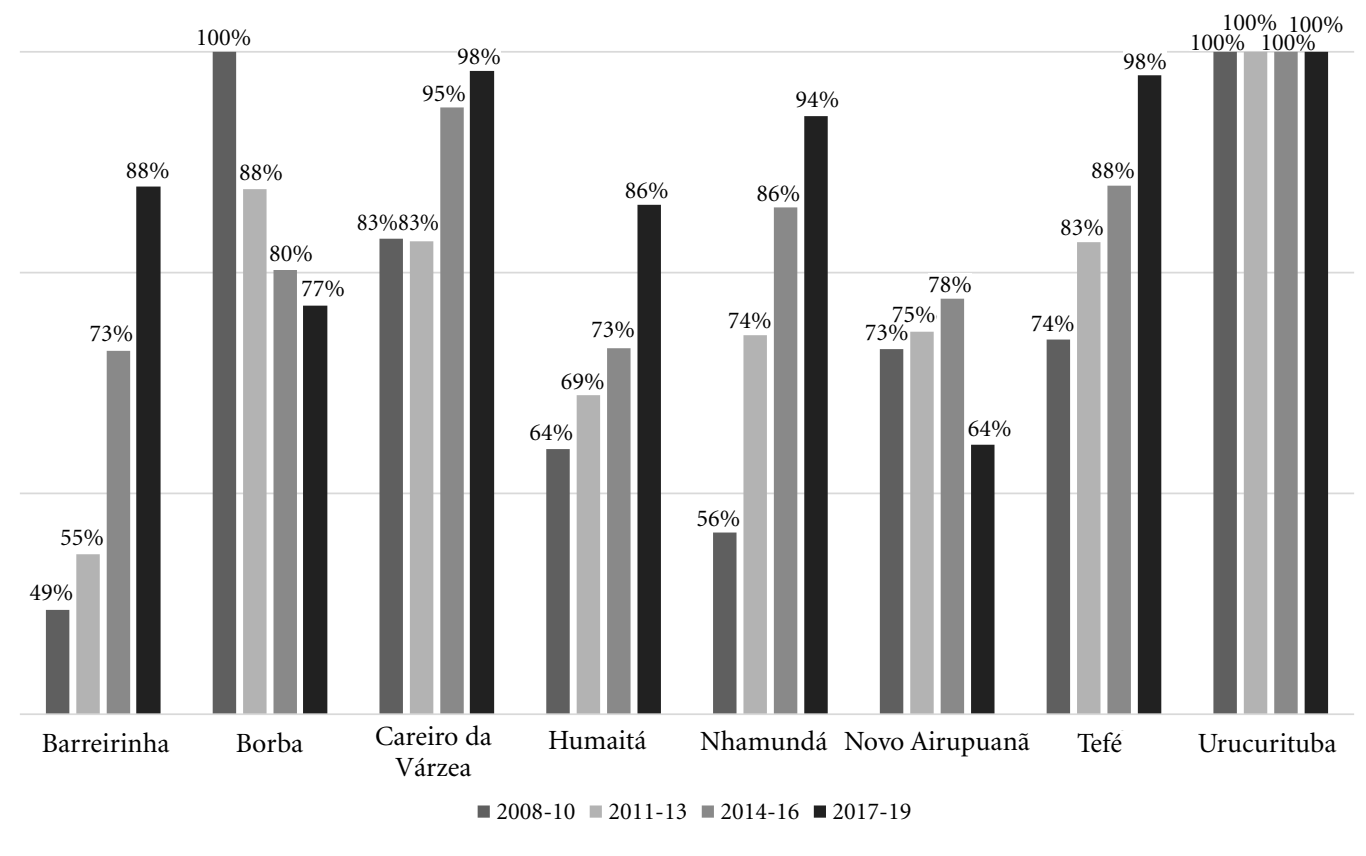

Figura 2. Evolução da cobertura da Atenção Básica nos municípios da amostra em triênios de 2008 a 2019.

Fonte: SISAB.

território amazônico na relação com as políticas de saúde ${ }^{8,9}$. O território na Amazônia é profundamente marcado pelo regime das águas e pelo ciclo hidrológico, por isso a necessidade de incorporar nas análises a dimensão desse território líquido que interfere e dialoga com os modos de vida da população. Assim, a ideia de um serviço móvel, em detrimento do fixo, ou de um fixo que se torna fluxo, conforme as categorias de Milton Santos ${ }^{17}$, representa uma grande mudança nos modos de agir sobre o lugar. O caminho das águas e das pessoas moldam os processos de trabalho e os serviços de saúde ${ }^{8,16}$. Assim, o território líquido se torna um analisador para olhar e interpretar as políticas públicas, nos apresentam informações sobre as populações ribeirinhas e seus modos de vida que subsidiam as políticas públicas. Conhecer o território, o lugar e as pessoas é um dos pressupostos para qualquer ação e planejamento em saúde, que permita evidenciar a expansão da cobertura de $\mathrm{AB}$ nessas áreas, como expressão do fortalecimento da política pública viável, sustentável e adequada.

Os resultados sobre a produção das equipes nos conduzem para a discussão sobre os processos de trabalho nesse tipo de território, pois ainda é objeto de discussão de gestores que estão em processo de implantação/credenciamento da política de saúde fluvial e ribeirinha. A dificuldade é pensar no tipo de contrato de trabalho das equipes que necessitam permanecer um tempo contínuo e longo nas embarcações ${ }^{5,9,16}$. Os contratos de trabalho se espelharam na modalidade da saúde indígena que utiliza o formato de 20 dias de trabalho em área e 10 de folga ${ }^{18}$. Esse formato traz como consequência a rotatividade dos profissionais de saúde, perdendo uma das características da $\mathrm{AB}$ que é a criação de vínculo entre o profissional e o usuário ${ }^{19}$.

Os resultados mostraram que não há muita diferença entre o trabalho das equipes ribeirinhas com as que tem características urbanas, com exceção dos técnicos e auxiliares de enfermagem que se destacam na produção. As equipes de contexto ribeirinho e fluvial tem um processo de trabalho mais colaborativo e interprofissional ${ }^{5,16}$, decorrente do convívio e da intensidade das relações. O trabalho em áreas rurais traz um relativo isolamento da equipe, mas isso pode contribuir por um trabalho interdisciplinar ${ }^{20}$. Assim, a participação de todos os profissionais no cuidado aumenta o escopo das práticas de saúde, onde a 
Tabela 2. Ações e produções das ESF nos municípios selecionados, conforme área de atuação, Amazonas, 2019.

\begin{tabular}{|c|c|c|c|c|c|c|}
\hline \multicolumn{7}{|c|}{ Municípios Urbanos $^{\mathrm{a}}$} \\
\hline & \multicolumn{2}{|r|}{ ESF Urbana } & \multicolumn{2}{|c|}{ ESF Fluvial } & \multicolumn{2}{|r|}{ ESF Ribeirinha } \\
\hline & Média & $(\mathrm{IC} 95 \%)$ & Média & $(\mathrm{IC} 95 \%)$ & Média & $(\mathrm{IC} 95 \%)$ \\
\hline \multicolumn{7}{|l|}{ Ações Individuais } \\
\hline ACS & 2243,1 & $0,777(0,608-0,945)$ & 1718,4 & $0,389(0,199-0,58)$ & 2160,6 & $2,221(1,538-2,903)$ \\
\hline TEC/AUX ENF & 495,8 & $0,172(0,138-0,205)$ & 1606,8 & $0,364(0,062-0,666)$ & 1032,0 & $1,061(0,513-1,608)$ \\
\hline ENF & 135,3 & $0,047(0,041-0,052)$ & 163,4 & $0,037(0,025-0,049)$ & 146,0 & $0,15(0,097-0,203)$ \\
\hline MED & 318,4 & $0,11(0,098-0,123)$ & 202,9 & $0,046(0,018-0,074)$ & 136,2 & $0,14(0,097-0,183)$ \\
\hline \multicolumn{7}{|l|}{ Ações Coletivas } \\
\hline ACS & 11,9 & $0,005(0,003-0,007)$ & 17,3 & $0,011(-)$ & 3,2 & $0,001(0,001-0,002)$ \\
\hline TEC/AUX ENF & 6,0 & $0,001(0,001-0,002)$ & 26,0 & $0,016(-)$ & 11,2 & $0,004(0,000-0,008)$ \\
\hline ENF & 11,0 & $0,004(0,003-0,005)$ & 6,8 & $0,004(-)$ & 8,3 & $0,009(0,003-0,014)$ \\
\hline MED & 1,7 & $0,001(0,001-0,001)$ & - & - & 5,6 & $0,006(0,000-0,012)$ \\
\hline \multicolumn{7}{|c|}{ Municípios Rurais $^{\mathrm{b}}$} \\
\hline & \multicolumn{2}{|r|}{ ESF Urbana } & \multicolumn{2}{|c|}{ ESF Fluvial } & \multicolumn{2}{|r|}{ ESF Ribeirinha } \\
\hline & Média & Razão (IC95\%) & Média & Razão (IC95\%) & Média & Razão (IC95\%) \\
\hline \multicolumn{7}{|l|}{ Ações Individuais } \\
\hline ACS & 855,0 & $0,647(0,507-0,786)$ & 835,0 & $0,227(0,106-0,349)$ & 869,2 & $0,464(0,332-0,595)$ \\
\hline TEC/AUX ENF & 179,6 & $0,052(0,038-0,065)$ & 296,9 & $0,081(0,000-0,177)$ & 473,6 & $0,253(0,17-0,336)$ \\
\hline ENF & 93,0 & $0,07(0,056-0,084)$ & 115,5 & $0,031(0,013-0,05)$ & 111,0 & $0,059(0,042-0,077)$ \\
\hline MED & 182,5 & $0,053(0,043-0,062)$ & 151,3 & $0,041(0,028-0,054)$ & 145,8 & $0,078(0,063-0,092)$ \\
\hline \multicolumn{7}{|l|}{ Ações Coletivas } \\
\hline ACS & 6,6 & $0,005(0,003-0,007)$ & 10,2 & $0,003(-)$ & 4,3 & $0,002(0,001-0,004)$ \\
\hline TEC/AUX ENF & 3,6 & $0,002(0,001-0,002)$ & 2,8 & $0,001(0,000-0,002)$ & 1,7 & $0,003(0,002-0,004)$ \\
\hline ENF & 7,4 & $0,002(0,002-0,003)$ & 9,0 & $0,002(0,000-0,006)$ & 6,7 & $0,004(0,002-0,005)$ \\
\hline MED & 2,3 & $0,001(0,001-0,002)$ & - & - & 3,0 & $0,001(0,001-0,002)$ \\
\hline \multicolumn{7}{|c|}{ Total de Municípios } \\
\hline & \multicolumn{2}{|r|}{ ESF Urbana } & \multicolumn{2}{|r|}{ ESF Fluvial } & \multicolumn{2}{|r|}{ ESF Ribeirinha } \\
\hline & Média & Razão (IC95\%) & Média & Razão (IC95\%) & Média & Razão (IC95\%) \\
\hline \multicolumn{7}{|l|}{ Ações Individuais } \\
\hline ACS & 1587,6 & $1,201(0,963-1,438)$ & 1087,4 & $0,296(0,165-0,427)$ & 1180,9 & $0,63(0,462-0,798)$ \\
\hline TEC/AUX ENF & 375,8 & $0,109(0,087-0,13)$ & 733,5 & $0,20(0,015-0,385)$ & 633,1 & $0,338(0,228-0,448)$ \\
\hline ENF & 115,7 & $0,087(0,078-0,097)$ & 129,2 & $0,035(0,022-0,049)$ & 120,2 & $0,064(0,049-0,079)$ \\
\hline MED & 265,0 & $0,077(0,068-0,085)$ & 166,8 & $0,045(0,032-0,059)$ & 143,1 & $0,076(0,064-0,088)$ \\
\hline \multicolumn{7}{|l|}{ Ações Coletivas } \\
\hline ACS & 9,9 & $0,007(0,005-0,01)$ & 13,7 & $0,004(0,002-0,006)$ & 3,9 & $0,002(0,001-0,003)$ \\
\hline TEC/AUX ENF & 5,1 & $0,002(0,001-0,003)$ & 10,5 & $0,004(0,000-0,01)$ & 7,1 & $0,011(0,000-0,022)$ \\
\hline ENF & 9,3 & $0,003(0,002-0,003)$ & 8,5 & $0,002(0,000-0,005)$ & 7,2 & $0,004(0,003-0,005)$ \\
\hline MED & 2,0 & $0,001(0,001-0,001)$ & & $(-)$ & 3,9 & $0,002(0,001-0,003)$ \\
\hline Classificação confor & tipologia & do IBGE, sendo agrupado 1 & Caleg & "municípios rurais" aquel & es que nãc & $\begin{array}{l}\text { eram urbanos. Municípios } \\
\text { lanã, Urucurituba. Nota 1: } \\
\text { iros; MED: Médicos; ESF: }\end{array}$ \\
\hline
\end{tabular}

Fonte: SISAB.

participação de todos os profissionais no cuidado aumenta o escopo das práticas de saúde.

$\mathrm{O}$ processo de trabalho nas equipes ribeirinhas e fluviais se constituem em outra dinâmica devido à existência de uma equipe ampliada, 
Tabela 3. Perfil das ESF nos municípios selecionados, conforme Indicadores do Previne e área de atuação, Amazonas, 2019.

\begin{tabular}{|c|c|c|c|c|c|c|}
\hline & \multicolumn{6}{|c|}{ Municípios Urbanos ${ }^{\mathrm{a}}$} \\
\hline & \multicolumn{2}{|c|}{ ESF Urbana } & \multicolumn{2}{|c|}{ ESF Fluvial } & \multicolumn{2}{|c|}{ ESF Ribeirinha } \\
\hline & Média & (IC95\%) & Média & (IC95\%) & Média & (IC95\%) \\
\hline \multicolumn{7}{|l|}{ Indicador PREVINE (\%) } \\
\hline Pré-Natal (6 consultas) & 50,9 & $(43,8-58,0)$ & 18,5 & $(0-37,6)$ & 19,3 & $(0-66,1)$ \\
\hline Pré-Natal (Sífilis e HIV) & 61,8 & $(51,4-72,2)$ & 26,5 & $(7,4-45,6)$ & 38,3 & $(0-110,2)$ \\
\hline Gestantes Saúde Bucal & 52,0 & $(44,6-59,4)$ & 29,0 & $(0-79,8)$ & 27,0 & $(0-87,2)$ \\
\hline Cobertura Citopatológico & 25,1 & $(19,7-30,5)$ & 24,0 & $(0-87,5)$ & 9,7 & $(3,4-15,9)$ \\
\hline Hipertensão (PA Aferida) & 16,9 & $(14,5-19,3)$ & 11,5 & $(0-94,1)$ & 5,0 & $(0-17,9)$ \\
\hline \multirow[t]{4}{*}{ Diabetes (Hemog. Glicada) } & 13,8 & $(10,3-17,2)$ & 6,0 & $(0-31,4)$ & 1,3 & $(0-2,8)$ \\
\hline & \multicolumn{6}{|c|}{ Municípios Rurais ${ }^{\mathrm{b}}$} \\
\hline & \multicolumn{2}{|c|}{ ESF Urbana } & \multicolumn{2}{|c|}{ ESF Fluvial } & \multicolumn{2}{|c|}{ ESF Ribeirinha } \\
\hline & Média & (IC95\%) & Média & (IC95\%) & Média & (IC95\%) \\
\hline \multicolumn{7}{|l|}{ Indicador PREVINE (\%) } \\
\hline Pré-Natal (6 consultas) & 46,4 & $(36,7-56,0)$ & 25,0 & $(0-75,8)$ & 33,0 & $(0-109,2)$ \\
\hline Pré-Natal (Sífilis e HIV) & 64,3 & $(52,3-76,4)$ & 49,0 & $(0-125,2)$ & 45,0 & $(0-400,8)$ \\
\hline Gestantes Saúde Bucal & 50,5 & $(39,5-61,5)$ & 72,0 & $(46,6-97,4)$ & 17,5 & $(11,2-23,9)$ \\
\hline Cobertura Citopatológico & 32,8 & $(24,2-41,3)$ & 25,5 & $(0-57,3)$ & 23,5 & $(17,2-29,9)$ \\
\hline Hipertensão (PA Aferida) & 11,8 & $(4,8-18,7)$ & 2,5 & $(0-8,9)$ & 6,5 & $(0-76,4)$ \\
\hline \multirow[t]{4}{*}{ Diabetes (Hemog. Glicada) } & 11,2 & $(3,2-19,2)$ & - & - & 3,5 & $(0-9,9)$ \\
\hline & \multicolumn{6}{|c|}{ Total de Municípios } \\
\hline & \multicolumn{2}{|c|}{ ESF Urbana } & \multicolumn{2}{|c|}{ ESF Fluvial } & \multicolumn{2}{|c|}{ ESF Ribeirinha } \\
\hline & Média & (IC95\%) & Média & (IC95\%) & Média & (IC95\%) \\
\hline \multicolumn{7}{|l|}{ Indicador PREVINE (\%) } \\
\hline Pré-Natal (6 consultas) & 49,1 & $(43,6-54,6)$ & 21,8 & $(13,6-29,9)$ & 24,8 & $(5,1-44,5)$ \\
\hline Pré-Natal (Sífilis e HIV) & 62,8 & $(55,2-70,4)$ & 37,8 & $(15,6-59,9)$ & 41,0 & $(5,4-76,6)$ \\
\hline Gestantes Saúde Bucal & 51,4 & $(45,5-57,4)$ & 50,5 & $(10,6-90,4)$ & 23,2 & $(1,0-45,5)$ \\
\hline Cobertura Citopatológico & 28,1 & $(23,5-32,7)$ & 23,8 & $(17,2-30,3)$ & 16,0 & $(4,8-27,2)$ \\
\hline Hipertensão (PA Aferida) & 14,9 & $(11,9-17,9)$ & 7,0 & $(0-18,8)$ & 5,6 & $(0-12,3)$ \\
\hline Diabetes (Hemog. Glicada) & 12,8 & $(9,2-16,3)$ & 3,5 & $(0-8,8)$ & 2,2 & $(0,6-3,8)$ \\
\hline
\end{tabular}

a,b Municípios urbanos: Tefé e Humaitá. Municípios rurais: Barreirinha, Borba, Careiro da Várzea, Nhamundá, Novo Aripuanã, Urucurituba. Nota 1: Usado maior resultado do indicador Previne dentre os quadrimestres de 2019 e primeiro de 2020. Nota 2: Quando o limite inferior do Intervalo de 95\% de Confiança (IC95\%) foi um valor negativo, utilizamos o zero " 0 ” na tabela.

Fonte: SISAB.

são de apoio e de logística, mas há situações em que os profissionais de saúde precisam saber de mecânica de motor de barco, conhecer os caminhos e canais nos rios ${ }^{18,21}$. No caso de áreas de acesso por estrada, o motorista também faz parte do trabalho da equipe ${ }^{22}$.

A enfermagem tem um papel de destaque nas equipes ${ }^{16}$, pois é responsável pela logística de alimentação, insumos, combustível, preparar a escala de trabalho, além de ser responsável pelo cuidado em saúde. Significa dizer que na Amazônia o processo de trabalho em saúde, seja na saúde indígena ou na saúde fluvial/ribeirinha, é ampliado, exigindo dos profissionais competências, habilidades e atitudes que não são avaliadas, ou "medidas", pelas métricas de produção. O trabalho das equipes de saúde rural precisa ter uma maior resolutividade devido a menor frequência dos atendimentos, longas distâncias a percorrer e a dispersão da população ${ }^{22}$. Isso indica a necessidade de compreender melhor as ações da $\mathrm{AB}$ no contexto da Amazônia, bem como a necessidade de avaliar políticas de saúde direcionadas para os modos de vida e as condições de saúde das populações rurais ${ }^{23,24}$.

Outro componente do trabalho nas áreas ribeirinhas é o tempo de deslocamento da equipe, que precisa ser incorporado ao tempo de trabalho, pois na Amazônia o tempo é a medida para os fluxos no território ${ }^{25,26}$. No caso da UBS fluvial, 
a casa e o trabalho se confundem, pois os trabalhadores descansam e vivem no local de trabalho, e, enquanto dormem, se deslocam para outra comunidade. Em algumas equipes ribeirinhas com características urbanas, como é o caso do município de Tefé, precisa se deslocar diariamente até sua área de trabalho, o que pode representar horas numa lancha, e a necessidade de carregar todos os insumos utilizados para a ação do dia. Nesse caso, observa-se que a organização do processo de trabalho acompanha as especificidades do território ${ }^{20}$.

O ACS, como morador da Comunidade onde atua, tem a função de fazer a articulação entre a população adscrita e as equipes ribeirinhas e fluviais $^{16,27,28}$, principalmente nos intervalos entre uma viagem e outra da UBSF, que pode representar de um a dois meses, dependendo o período do $a^{5} o^{5}$ e da cheia dos rios. Estudo realizado numa Comunidade quilombola e ribeirinha na região do Baixo Rio Amazonas, mostrou que o ACS realiza uma atenção para além do que está preconizado pelo MS, pois articula outros saberes e práticas que fazem parte da memória e história da comunidade ${ }^{29}$. Assim, a análise da produção desse profissional pode esconder as práticas e saberes que permanecem na sombra dos registros oficiais.

A Saúde da Família, desde sua criação em 1994, se colocou como a principal estratégia de cunho universal para a $\mathrm{AB}$, tendo o princípio da equidade como forma de promoção de uma atenção diferenciada para populações específicas $^{15,30}$. A expansão das ESF foi acompanhada de outras equipes, como o Consultório na Rua, Núcleo Ampliado da Saúde da Família (NASF-AB), equipes de Saúde Ribeirinha (eSFR) e equipes de Saúde Fluvial (eSFF) ${ }^{7,15}$ e, com outros Programas, como o Mais Médicos, significando uma cobertura de $63.7 \%$ da população ${ }^{2}$. Desse modo, as equipes analisadas no presente estudo têm um impacto importante sobre a qualidade de vida da população rural amazônica, mas há limitação no dimensionamento da atenção quando se utiliza indicadores e dados disponíveis nos sistemas de informação, sendo, portanto, necessário a realização de estudos de natureza qualitativa e com metodologias participativas com as equipes e comunidade ${ }^{31}$.

Estudos mostram que "quanto maior a cobertura, menor é o número de internações por causas sensíveis à atenção básica" ${ }^{2,3}$. A presença da equipe nos territórios ribeirinhos e rurais significa a realização de ações programáticas que contribuem para um cuidado preventivo e a qualidade de vida da população, algo inédito na história da Amazônia, que é marcada por ações campanhistas, que limitam a integralidade do cuidado $^{5,8,9,16}$. E aqui vale salientar que, mais do que a presença das equipes neste território, parece haver uma qualidade similar em grande parte das ações entre os tipos de equipes, sobretudo quando exigido pouco acesso à tecnologia médica, pois o desempenho foi similar entre equipes rurais e urbanas nos indicadores do Previne referentes à pré-natal (sífilis e HIV), saúde bucal de gestantes, cobertura em exame citopatológico e acompanhamento de PA entre hipertensos.

É compreensível o maior desempenho entre ESF urbanas para alcançar o mínimo de seis consultas de pré-natal quando comparados às equipes fluviais, devido esta possuir característica itinerante e, portanto, menor capacidade de longitudinalidade do cuidado. Igualmente, já era esperado uma maior média de exames de hemoglobina glicada nas equipes urbanas em relação às equipes fluviais e ribeirinhas, uma vez que na área rural destes municípios há maior dificuldade de acesso a laboratórios. Pereira e Pacheco ${ }^{32}$ estudando o Programa Mais Médicos na atenção à saúde em município predominantemente rural no estado do Pará sugerem que o programa resultou em ampliação do acesso aos serviços de $\mathrm{AB}$ nessas áreas. No entanto, desvelou a continuidade de problemas recorrentes e que são externos à implantação de equipes de $\mathrm{AB}$ no território, como: falta de acesso a medicamentos e exames, limitações no retorno dos pacientes referidos a outros serviços e fragilidades na rede de atenção à saúde; o que prejudica diretamente a busca da resolutividade e integralidade do cuidado.

Estudos oriundos do Programa Nacional de Melhoria do Acesso e da Qualidade da Atenção Básica (PMAQ) revelam certa similaridade com os nossos achados. A avaliação de equipes de $\mathrm{AB}$ do município de Montes Claros-MG pelos profissionais, em 2014, não encontrou diferença significativa entre equipes urbanas e rurais nas dimensões educação permanente, processo de trabalho e atenção integral à saúde, de maneira geral, porém, as características de ruralidade deste município são bem diferentes daquelas encontradas entre os ribeirinhos da Amazônia ${ }^{24}$. Desta forma, mais especificamente na região norte do Brasil, Garnelo et al. ${ }^{33}$, avaliando acesso e cobertura da atenção primária em populações rurais e urbanas a partir de dados do segundo ciclo do PMAQ, identificaram que, de maneira geral, os usuários de origem rural tem mais dificuldades de acesso por conta do horário de funcionamen- 
to da unidade e do tempo de espera das visitas domiciliares. As visitas em áreas de difícil acesso necessitam de uma logística complexa, desde o tipo de transporte, combustível, domínio do ambiente e das mudanças ocasionadas pelo ciclo hidrológico.

Comparando com os de área urbana, os usuários de área rural se mostraram mais satisfeitos com o serviço de AB, e no estado do Amazonas, em particular, relataram que o horário de funcionamento atende suas necessidades, e, quando precisam de consulta, são geralmente bem atendidos, percebendo alguma ação para resolução de seus problemas, além de receberem mais visitas de ACS. Sobre o nível de satisfação, os autores do estudo trazem que tal percepção pode ter sido influenciada pela disponibilização de ações de saúde, comparada ao vazio assistencial anterior à implantação do modelo da ESF, mesmo frente a barreiras observadas por atores externos às comunidades, como os profissionais ${ }^{33}$. Assim, ainda no Amazonas, não houve muitas diferenças na qualidade de atenção e serviço prestados entre equipes de $\mathrm{AB}$ urbanas e rurais, pela visão das equipes, corroborando com o indicado pelos resultados do Previne analisados.

O presente estudo tem limite quando avalia as ações de promoção da saúde, porque não são facilmente identificadas na produção coletiva da equipe, que direciona suas ações para o componente de educação em saúde e que, na maioria das vezes, são traduzidos em ações como palestras sobre prevenção às doenças que compõem o calendário de campanhas e outras específicas da região amazônica. Assim, há necessidade de aprofundar os estudos que analisem tanto os processos de educação permanente em saúde como formas de fazer a promoção de saúde nos territórios líquidos da Amazônia. Por fim, objetivando maior qualidade de comparação, tais análises devem ser objeto de futuros estudos, quando houver maior número de ESF ribeirinhas implantadas na região.

Sabe-se também que a produção em serviços de saúde pode sofrer efeito de períodos do ano ou de locais diferentes. Neste sentido, optou-se pela por trabalhar com a média da produção em todo o período, a fim de reduzir a influência de meses com extremos de notificações, e ao agrupar municípios com características de urbanização e ruralidade amazônica semelhantes tentou-se levantar algumas hipóteses de efeito da localidade sobre a questão. Outro aspecto a ser considerado é a classificação dos municípios feita pelo IBGE, utilizada pelo MS, para caracterizar o urbano e o rural, e suas variações, onde Tefé, por exemplo, está categorizado como "urbano", mas tem uma significativa população rural e ribeirinha, com quase 200 comunidades dispersas num grande território, que demanda um investimento considerável na atenção a essas famílias. O município possui uma ESFF e 4 ESFR, do total de 19 equipes $^{34}$.

\section{Considerações finais}

Este estudo aponta para o aumento da cobertura da atenção básica na maioria dos municípios investigados, sobretudo no último triênio, e que as iniciativas de criação de ESF nas áreas rurais, unidades fluviais ou ribeirinhas, tem otimizado o acesso de grande parte desta população aos serviços de saúde, apesar de ainda insuficientes para toda a população. Revela ainda que houve uma diferenciação das produções ambulatoriais entre equipes, segundo o tipo de território, o que denota possíveis adaptações em processos de trabalhos entre unidades rurais, com valorização dos papéis dos técnicos ou auxiliares de enfermagem e agentes comunitários de saúde (ACS).

Dentre os 61 municípios do interior do estado, apenas 8 tinham simultaneamente equipes de saúde fluvial e ribeirinha. A baixa adesão dos municípios para uma política que amplia o acesso das populações ribeirinhas, significando mais recursos para o custeio dessas equipes, com contratação de profissionais e apoio logístico, é algo que ainda precisa ser investigado e explicado. A ampliação desse tipo de política é uma forma de produzir justiça social para as populações ribeirinhas $^{20,34}$.

A avaliação e o monitoramento de modelos de fazer a gestão à saúde na Amazônia necessita ser pensado a partir das seguintes dimensões: tipos de equipe, população, território, fluxos e fixos, ciclo das águas, questões logísticas. A AB valoriza a dinâmica e o conhecimento dos territórios e da população, porque quanto mais próxima da realidade mais eficiência e eficácia haverá nos cuidados em saúde. Assim, os modelos de gestão do cuidado que estão em processo na Amazônia precisam ser ampliados, apoiados e incentivados a partir de processos de educação que priorizem políticas de acesso às populações das águas e da floresta.

A discussão de acesso precisa ser ampliada para além da oferta de equipamentos e equipes num determinado território ${ }^{35}$, necessitando pensar em estratégias participativas que envolvam as questões culturais, ambientais, étnicas e territo- 
riais. $\mathrm{O}$ trabalho em saúde em áreas ribeirinhas traz desafios importantes para a produção do acesso e da equidade, sendo que o argumento geográfico não pode ser utilizado para justificar a falta de investimentos e as dificuldades de gestão, pois é possível a criação e implementação de modelos tecnoassistenciais que cheguem até a porta das pessoas, seja pelos rios, estradas ou por outras trilhas que geram o encontro do cuidado.

A política de acesso à $\mathrm{AB}$, voltada para populações ribeirinhas, não apresenta como obstáculos as especificidades do território e sua cultura, mas, se constituem como desafios para uma saúde integral e equitativa das populações nos vários territórios da Amazônia. A pandemia da Covid-19 mostrou que a $\mathrm{AB}$ é a principal estra- tégia de enfrentamento, pois atua nos territórios de vida das pessoas. Assim, os ribeirinhos, indígenas, quilombolas e os povos da floresta importam e tem direito à atenção e cuidados diferenciados.

\section{Colaboradores}

RTS Lima, TG Fernandes, JC Schweickardt, CS Portela e PJA Martins Júnior participaram da concepção, revisão e redação final do manuscrito. JDO Santos Junior participou da análise estatística dos dados.

\section{Referências}

1. Brasil. Portaria no 2.488 , de 21 de outubro de 2011 . Política Nacional de Atenção Básica. Diário Oficial da União 2011; 21 out.

2. Cecilio LCO, Reis AAC. Apontamentos sobre os desafios (ainda) atuais da atenção básica à saúde. Cad Saude Publica 2018; 34(8):1-14.

3. Brasil. Portaria $\mathrm{n}^{\circ}$ 2.436, de 21 de setembro de 2017. Política Nacional de Atenção Básica. Diário Oficial da União 2017; 21 set.

4. Paim J, Travassos C, Almeida C, Bahia L, Macinko J. The Brazilian health system: history, advances, and challenges. Lancet 2011; 377(9779):1778-1797.

5. Lima RTS, Simões AL, Heufemann NE, Alves VP. Saúde sobre as águas: o caso da Unidade Básica de Saúde Fluvial. In: Intensidade na Atenção Básica: prospecção de experiências informes e pesquisa-formação. Porto Alegre: Rede Unida; 2016. p. 271-293.

6. Rios-Villamizar EA, Piedade MTF, Junk W. Tipologias de águas em áreas úmidas da Bacia Amazônica: uma revisão enfatizando a classificação dos rios e igarapés. In: Ferreira SJF, Silva ML, Pascoaloto D. Amazônia das águas: qualidade ecologia e educação ambiental. $\mathrm{Ma}$ naus: Valer, Fapeam; 2016.

7. Almeida ER, Brandão CC, Matielo E, Santana MA, Ugarte OA, Costa TS. Atenção Básica à Saúde: avanços e desafios no contexto amazônico. In: Schweickardt JC, Kadri MR El, Lima RTS, organizadores. Atenção Básica na Região Amazônica: saberes e práticas para o fortalecimento do SUS. Porto Alegre; 2019. p. 15-50.
8. Schweickardt JC, Lima RTSL, Simões AL, Freitas CM, Marques VP. Território na Atenção Básica: abordagem da Amazônia equidistante. In: In-formes da Atenção Básica: aprendizados de intensidade por círculos em rede. Porto Alegre: Rede Unida; 2016. p. 101-131.

9. Kadri RM, Santos BS, Lima RTS, Schweickardt JC, Martins FM. Unidade Básica de Saúde Fluvial: um novo modelo da Atenção Básica para a Amazônia, Brasil. Interface (Botucatu) 2019; 23:e180613.

10. Brasil. Ministério da Saúde (MS). Secretaria de Atenção Básica. Sistema de Informação em Saúde para a Atenção Básica-SISAB: Nota técnica - Relatório de Envio: produção enviada [Internet]. Brasília: MS; 2019. Disponível em: http://189.28.128.100/dab/docs/portaldab/notas_tecnicas/nt_envio_producao_012016. pdf

11. Instituto Brasileiro de Geografia e Estatística (IBGE). Atlas do censo demográfico 2010. Rio de Janeiro: IBGE; 2013.

12. Brasil. Ministério da Saúde (MS). Secretaria de Atenção Básica. Portaria no 2.979, de 12 de novembro de 2019. Institui o Programa Previne Brasil, que estabelece novo modelo de financiamento de custeio da Atenção Primária à Saúde no âmbito do Sistema Único de Saúde, por meio da alteração da Portaria de Consolidação $n^{\circ}$ 6/GM/MS, de 28 de setembro de 2017. Diário Oficial da União 2019; 13 nov. 
13. Instituto Brasileiro de Geografia e Estatística (IBGE). Classificação e caracterização dos espaços rurais e urbanos do Brasil: uma primeira aproximação. Rio de Janeiro: IBGE; 2017.

14. Pinto H. Análise da mudança da política nacional de atenção básica. Saude Redes 2018; 4(2):191-217.

15. Sousa MF, Hamann EM. Programa Saúde da Família no Brasil: uma agenda incompleta? Cien Saude Colet 2009; 14(Supl. 1):1325-1335.

16. Figueira MC, Marques D, Vilela MF, Pereira JA, Bazílio J, Silva EM. Fluvial family health: work process of teams in riverside communities of the Brazilian Amazon. Rural Remote Health 2020; 20(3):5522.

17. Santos M. Técnica, Espaço, Tempo: Globalização e meio técnico-científico informacional. São Paulo: Hucitec; 1978.

18. Schweickardt JC, Ferla AA, Lima RTS, Amorim JSC. O Programa Mais Médicos na saúde indígena: o caso do Alto Solimões, Amazonas, Brasil. Rev Panam Salud Publica 2020; 44:e24.

19. Starfield B. Atenção primária: equilíbrio entre necessidades de saúde, serviços e tecnologia. Brasília: ONU, MS; 2002.

20. Savassi LCM, Almeida MM, Floss M, Lima MC. Saúde no caminho da roça. Rio de Janeiro: Fiocruz; 2019.

21. Schweickardt JC, Fela AA, Silva JMBF, Lima RTS, Andrade TR, Caldas JN, Ahmadpour B. O Programa Mais Médicos e seus encontros com o cuidado indígena no DSEI Alto Rio Solimões: anotações do campo de pesquisa. In: Schweickardt JC, Silva JMBF, Ahmadpour B. Saúde indígena: práticas e saberes por um diálogo intercultural. Porto Alegre: Editora Rede Unida; 2020.

22. Lima MC, Teixeira ACA, Barros E, Savassi LCM, Almeida MM, Segura MC, Anderson MIP. O fazer rural. In: Savassi LCM, Almeida MM, Floss M, Lima MC. Saúde no caminho da roça. Rio de Janeiro: Fiocruz; 2019.

23. Coimbra CEA. Saúde Rural no Brasil: tema antigo mais que atual. Rev Saude Publica 2018; 52(Supl. 1):2s.

24. Moreira KS, Vieira MA, Costa SM. Qualidade da Atenção Básica: avaliação das Equipes de Saúde da Família. Saude Debate 2016; 40(111):117-127.

25. Ferla AA, Trepte RF, Schweickardt JC, Lima RTS, Martino A. Os (des)encontros da pesquisa no tempo-espaço amazônico: um ensaio sobre produção de conhecimento como abertura à aprendizagem. Rev Saude Redes 2016; 3:241-261.

26. Kadri MR, Freitas CM. Um SUS para Amazônia: contribuições do pensamento de Boaventura de Sousa Santos. Cien Saude Colet 2019 (no prelo). Disponível em: http://www.cienciaesaudecoletiva. com.br/artigos/um-sus-para-amazonia-contribuicoes-do-pensamento-de-boaventura-de-sousa-santos $/ 17453$ ?id $=17453$.

27. Pereira FR, Schweickardt JC, Lima RTS, Schweickardt KHSC. O banzeiro no território líquido da Amazônia: a micropolítica do trabalho de uma equipe de saúde ribeirinha. In: Schweickardt JC, Kadri MR, Lima RTS, organizadores. Atenção Básica na região Amazônica: saberes e práticas para o fortalecimento do SUS. Porto Alegre: Rede Unida; 2019. p. 92-111.
28. Santos BS. Micropolítica da gestão do trabalho e do cuidado na UBS fluvial de Borba-AM [dissertação]. Manaus: Programa de Pós-Graduação em Condição de vida e Situações de Saúde na Amazônia, Fiocruz Amazônia; 2018.

29. Silva JMBF, Schweickardt JC, Ferla AA, Heufemann NEC. Saúde numa comunidade quilombola da Amazônia. In: Schweickardt JC, Kadri MR, Lima RTS, organizadores. Atenção Básica na região Amazônica: saberes e práticas para o fortalecimento do SUS. Porto Alegre: Rede Unida; 2019. p. 112-125.

30. Viana AL, Dal Poz MR. A reforma do Sistema de Saúde no Brasil e o Programa de Saúde da Família. Physis 1998; 8(2):11-48.

31. Giatti L. O caráter adaptativo da pesquisa participativa: rompendo com a monocultura de saberes. In: Toledo RF, Rosa TEC, Keinert TM, Cortizo CT, organizadores. Pesquisa Participativa em Saúde: vertentes e veredas. São Paulo: Instituto de Saúde; 2018.

32. Pereira LL, Pacheco L. O desafio do Programa Mais Médicos para o provimento e a garantia da atenção integral à saúde em áreas rurais na região amazônica, Brasil. Interface (Botucatu) 2017; 21(Supl. 1):11811192.

33. Garnelo L, Lima JG, Rocha ESC, Herkrath FJ. Acesso e cobertura da Atenção Primária à Saúde para populações rurais e urbanas na região norte do Brasil. Saude Debate 2018; 42(1):81-99.

34. Azevedo ES, Schweickardt JC, Martins FM, Lima NPT, Moreira MA. Saúde ribeirinha em Tefé: aprendizados da atenção básica em um território em movimento. In: Moreira MA, Figueiró R, Ferla AA, Schweickardt JC. Educação permanente em saúde em Tefé-AM: qualificação do trabalho no balanço do banzeiro. Porto Alegre: Rede Unida; 2019.

35. Sousa ABL, Monteiro IOP, Bousquat A. Atenção Primária à Saúde em áreas rurais Amazônicas: análise a partir do planejamento do distrito de saúde rural de Manaus. In: Schweickardt JC, Kadri MR, Lima RTS, organizadores. Atenção Básica na região Amazônica: saberes e práticas para o fortalecimento do SUS. Porto Alegre: Rede Unida, 2019. p. 71-91.

Artigo apresentado em 21/10/2020

Aprovado em 02/02/2021

Versão final apresentada em 04/02/2021

Editores-chefes: Maria Cecília de Souza Minayo, Romeu Gomes, Antônio Augusto Moura da Silva 